\title{
Targeting COX-2 potently inhibits proliferation of cancer cells in vivo but not in vitro in cutaneous squamous cell carcinoma
}

\author{
Lipeng Gao ${ }^{1 \#}$, Tim Hua Wang ${ }^{1 \#}$, Champ Peng Chen ${ }^{2 \#}$, Jan Jian Xiang ${ }^{2}$, Xu-Bo Zhao ${ }^{1}$, Rong-Yin Gui ${ }^{1}$, \\ Xin-Hua Liao ${ }^{1 \wedge}$ \\ ${ }^{1}$ School of Life Sciences, Shanghai University, Shanghai, China; ${ }^{2}$ Fujian Key Laboratory for Translational Research in Cancer and Neurodegenerative \\ Diseases, Institute for Translational Medicine, Fujian Medical University, Fuzhou, China \\ Contributions: (I) Conception and design: XH Liao, CP Chen, JJ Xiang; (II) Administrative support: L Gao; (III) Provision of study materials or \\ patients: L Gao, TH Wang, CP Chen; (IV) Collection and assembly of data: L Gao, TH Wang, CP Chen, JJ Xiang, XB Zhao, RY Gui; (V) Data \\ analysis and interpretation: All authors; (VI) Manuscript writing: All authors; (VII) Final approval of manuscript: All authors. \\ \#These authors contributed equally to this work. \\ Correspondence to: Xin-Hua Liao. School of Life Sciences, Shanghai University, No. 333 Nanchen Road, Shanghai 200444, China. \\ Email: xinhualiao@foxmail.com.
}

Background: Cyclooxygenase 2 (COX-2) is an inducible enzyme which promotes tumorigenesis in many types of cancers. Genetic knockout of COX-2 significantly suppresses the tumorigenesis of skin squamous cell carcinoma (SCC). However, COX-2 inhibitor treatment only showed mild to moderate inhibition on SCC in previous reports. The aim of this study is to solve this contradiction and to re-evaluate the therapeutic potential of targeting COX-2 in SCC.

Methods: COX-2 was knocked down by shRNA in two different SCC cell lines, A431 and SCC-13. The cells proliferation and migration capacity were evaluated by cell growth curves and monolayer scratch assay, respectively. Cancer cells with COX-2 knockdown were also xenografted into Balb/c nude mice and tumor growth curves were recorded over time. In addition, we changed the drug administration route and intraperitoneally injected COX-2 inhibitor celecoxib into mice to evaluate its anti-cancer activity.

Results: Knockdown of COX-2 exhibited mild or even no effect on cell proliferation and migration in two different SCC cell lines in vitro. However, when cancer cells were xenografted into nude mice, knockdown of COX-2 significantly suppressed proliferation of cancer cells in tumors. At last, intraperitoneal injection instead of oral administration of COX-2 inhibitor celecoxib potently suppressed tumor growth.

Conclusions: Our results indicate that COX-2 might impact on the interaction between cancer cells and surrounding microenvironments rather than on cancer cells directly, and demonstrate that targeting COX-2 is a very promising therapeutic approach for SCC treatment.

Keywords: Prostaglandin-endoperoxide synthase 2 (PTGS2); skin cancer; celecoxib; cancer microenvironments; inflammation

Submitted Dec 29, 2020. Accepted for publication Mar 22, 2021.

doi: $10.21037 /$ tcr-20-3527

View this article at: http://dx.doi.org/10.21037/tcr-20-3527

\section{Introduction}

Cutaneous squamous cell carcinoma (SCC) is an atypical hyperplastic and aggressive cancer with a metastasis rate of about $0.1 \%$ to $9.9 \%$ (1). SCC is accounting for about $25 \%$ of non-melanoma skin cancer (NMSC) (2). There is still a lack of safe and effective medicines for treating malignant SCC.

Cyclooxygenase $(\mathrm{COX})$ is a rate-limiting enzyme in the reaction of arachidonic acid (AA) to prostaglandin

^ ORCID: 0000-0001-7520-7135. 
(PG). COX-2, also known as prostaglandin-endoperoxide synthase 2 (PTGS2), is an inducible enzyme (3). Under normal circumstances, COX-2 is rarely expressed in most cells, but its expression is significantly increased during inflammation (4). COX-2 in turn increases inflammation, forming a positive feedback loop, to promote tumorigenesis (5-7).

Previous studies have shown a strong expression of COX2 in skin exposed to sunlight and SCC (8-10). Transgenic overexpression of COX-2 increases the tumorigenesis of SCC (11). Consistently, genetic knockout of COX-2 significantly suppresses the tumorigenesis of SCC $(12,13)$. COX-2 inhibitors have been shown to suppress UV-induced SCC formation in albino hairless mice $(14,15)$. However, the hairless strains they used are uncharacterized/nonpedigreed. Therefore, the tumors from these mice showed considerable inter-individual variation. More importantly, they incorporated COX-2 inhibitor into food and the drug only exhibited mild to moderate anti-cancer activity $(14,15)$, which is inconsistent with the data of significant suppression of SCC tumorigenesis in COX-2 knockout mice $(12,13)$. As we know, oral administration may have potential limitations: lack of absorption from the digestive tract, degradation of substances by digestive enzymes and acid, and first-pass effect by the liver with reduced efficacy, etc. (16). Drugs are usually metabolized about 10 times faster in mice than in human if drug dosage is calculated based on body weight (17). Therefore, oral administration of drugs in mice could not fully represent the situation in human and usually reduce the efficacy of drugs as compared with other routes of drug administration like direct injection. Furthermore, although COX-2 has been extensively studied in skin cancer previously, how COX-2 protein directly functions onto skin cancer cells has not been examined.

In this study, to solve the contradiction of previous reports and to re-evaluate COX-2 as therapeutic target for human SCC treatment, we re-examined the effects of genetic and chemical inhibition of COX-2 on skin tumorigenesis in vitro and in vivo. We found that knockdown of COX-2 in two SCC cell lines exhibited slight or even no effects on proliferation and migration of cancer cells in vitro, but strongly inhibited their tumorigenesis using Balb/c nude mice xenograft tumor model, arguing that COX-2 might impact on the tumor microenvironments rather than on cancer cells directly. We also evaluated the anti-cancer activity of COX-2 inhibitor celecoxib on SCC tumorigenesis in vivo by intraperitoneal injection, and found it exerted very potent cancer inhibitory activity, which is now consistent with data from COX-2 knockout mice. Thus, our evidence demonstrates that COX-2 represents a very promising therapeutic target for human SCC treatment. We present the following article in accordance with the ARRIVE reporting checklist (available at http:// dx.doi.org/10.21037/tcr-20-3527).

\section{Methods}

\section{Ethical statement}

The study was conducted in accordance with the Declaration of Helsinki (as revised in 2013). Experiments were performed under a project license (No. 2019033) granted by the Experimental Animal Ethics Committee of Shanghai University, in compliance with the guidelines of the Institutional Animal Care and Use Committee (IACUC).

\section{Animals and cell lines}

Six-week-old Balb/c female nude mice with body weight $16 \pm 1 \mathrm{~g}$ were obtained from SLAC Ltd. (Shanghai, China). The animals were housed $<5$ per cage under SPF conditions, with free access to food and water under a $12 \mathrm{~h}$ light/dark cycle.

A431 cell line was obtained from Cell Bank of Chinese Academy of Sciences. SCC-13 cell line was a kind gift from Dr. James G. Rheinwald, Brigham and Women's Hospital and Harvard Skin Disease Research Center, USA $(16,18)$. These cells were cultured in low-calcium DMEM/F12 (3:1) supplemented with $10 \%$ fetal bovine serum (FBS, Gibco Co., USA) at $37{ }^{\circ} \mathrm{C}$ in a humidified atmosphere of $5 \% \mathrm{CO}_{2}$ and $95 \%$ air.

\section{COX-2 knockdown in cancer cells}

DNA oligos were synthesized and annealed to form a double-stranded internal hairpin DNA fragment targeting COX-2 sequence GCAGCTTCCTGATTCAAATGA (shRNA1) and the validated one GCAGATGAAATACCAG TCTTT (shRNA2) (19) with cohesive termini, which were then cloned into lentiviral plasmid pLKO.1-puro. The resulting plasmid was then transfected into 293 T cells together with helper plasmids pVSVG, pMDL and pREV. The virus were harvested and then incubated with cancer cells A431 or SCC-13 to infect them. Western blot analysis demonstrated that shRNA1 had much better knockdown 
efficacy on COX-2 than the validated shRNA2. shRNA1 was then used for all of following experiments.

\section{Scratch migration analysis}

A431 or SCC-13 cancer cells infected by lentivirus expressing scrambled or COX-2 shRNA were grown in 6-well plates with duplicate. When reaching a confluence of $\sim 95 \%$, cells were treated with mitomycin C to arrest growth. Two straight scratches were then made with a pipette tip across each cell monolayer. Total eight areas down through the two scratched lines were labeled on the bottom side of the plate by marker pen, and immediately recorded by camera; after 24 hours when the scratches almost closed, the same eight scratch areas were recorded again. The original and resulting areas absent of cells were quantified by ImageJ software. Normalization was relative to $\%$ control migration, set to 1.0 .

\section{Tumor xenografting and drug treatment}

$1 \times 10^{6} \mathrm{~A} 431$ or $2 \times 10^{6}$ SCC- 13 cells expressing scrambled or COX-2 shRNA together with matrigel (\#356231; BD) were inoculated subcutaneously onto the left and right flank region of 8-10 female Balb/c nude mice at the age of 6-week, respectively. Tumor volume was measured twice weekly and calculated by the formula $\left(\mathrm{L} \times \mathrm{W}^{2}\right) / 2$, where $\mathrm{L}$ and $\mathrm{W}$ were the lengths of the major and minor diameters, respectively.

Twenty days after A431 cancer cells inoculation, when average tumor volume reaches $\sim 35 \mathrm{~mm}^{3}, 20$ mice with similar total tumor volume were selected and divided into treatment and control groups. To avoid inefficient drug delivery by oral administration, the mice of treatment group and control group were injected intraperitoneally everyday with COX-2 inhibitor celecoxib or solvent only at dose of $5 \mathrm{mg} / \mathrm{kg}$ of body weight, respectively.

When tumor diameter reached $1.5 \mathrm{~cm}$, all of mice were euthanized and xenografted tumors were harvested.

\section{Tumor sample collection and analysis}

When tumor reached the endpoint, all mice were euthanized and tumors were dissected, photographed and weighed.

For western blot analysis, tumors were freezed and grounded in liquid nitrogen. The samples were dissolved in RIPA buffer with protease inhibitors and then sonicated and centrifuged. The supernatant was mixed with SDS loading buffer for further analysis. Protein samples $(20-35 \mu \mathrm{g})$ were resolved on $12 \%$ Tris-glycine gels and transferred onto a nitrocellulose membrane. After blocking the nonspecific binding sites, the membrane was incubated with the primary antibody at $4{ }^{\circ} \mathrm{C}$ overnight. The membrane was then incubated with appropriate horseradish peroxidaseconjugated secondary antibody and the immunoreactive bands were visualized using chemiluminescence reagent and the images were captured by CCD camera.

For immunofluorescence analysis, tumor samples were embedded and frozen in OCT compound (Sakura Finetek). Samples were then sectioned at 6-9 $\mu \mathrm{m}$. Sections were then fixed in 4\% PFA and stained with Alexa Fluor conjugated antibodies. Fluorescence images were visualized and captured by fluorescent microscopy.

Antibodies used in this study is listed as following: rabbit anti-COX-2 (\#12282S; Cell Signaling Technology), rabbit anti-Ki67 (\#AB15580; Abcam), rat anti-BrdU (\#AB6326; Abcam) and mouse anti-Actin (\#HC201; TransGen Biotech).

\section{Statistical analysis}

Statistical differences were evaluated by two-tailed student's $t$-test (GraphPad Prism v8.0, GraphPad Software, San Diego, CA, USA). The difference was considered to be significant when $\mathrm{P}<0.05\left(^{*}\right), \mathrm{P}<0.01\left(^{(*)}\right.$ and $\left.\mathrm{P}<0.001{ }^{(* *}\right)$.

\section{Results}

\section{COX-2 knockdown has no effect on proliferation and migration of A431 cells in vitro}

Although COX-2 has been extensively studied and shown to significantly impact on SCC tumorigenesis, the mechanism of how COX-2 conducts its functions in SCC has not been investigated. To examine whether COX-2 directly impacts on cancer cells, we knocked down COX-2 expression in SCC cancer cell line A431 by shRNA. Western blot results demonstrated an obvious reduction of COX-2 expression in cancer cells infected with lentivirus expressing COX-2 shRNA (Figure 1A). Cell growth was not significantly affected after COX-2 knockdown, as illustrated by growth curves over time (Figure 1B). COX-2 knockdown also had no effect on A431 cell migration, as illustrated by statistical results of cell monolayer scratch assay (Figure 1C,D). Overall cell morphology of A431 didn't change as well (data not shown). 
A

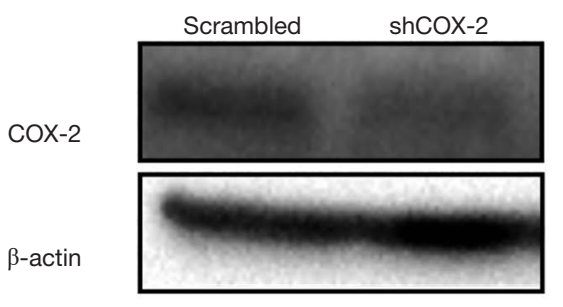

B

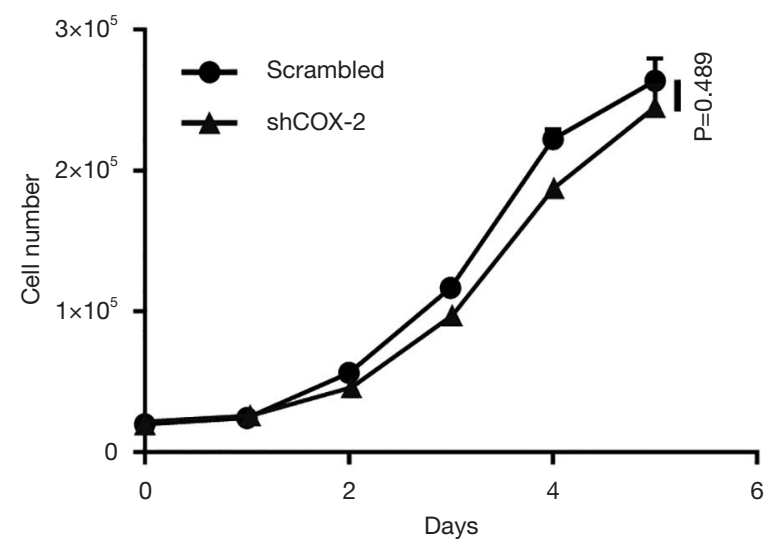

C
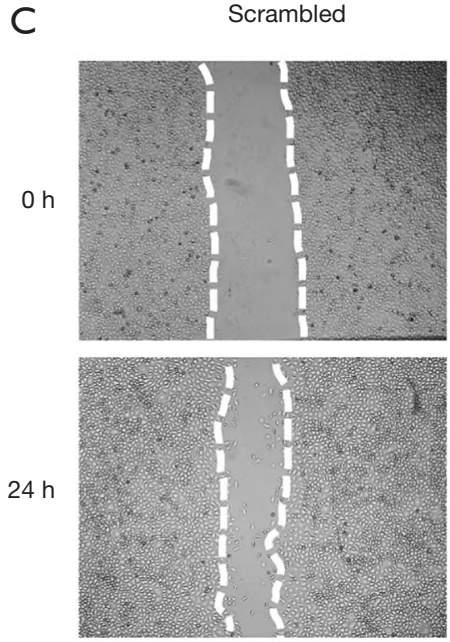

shCOX-2
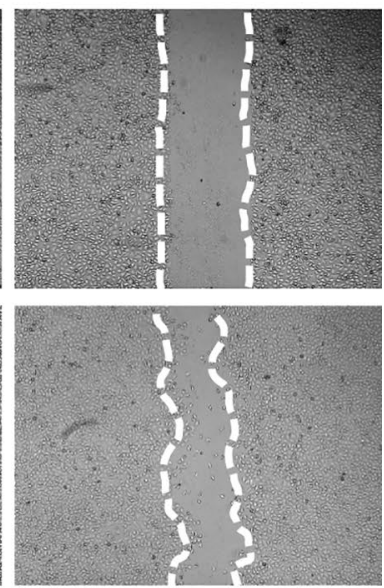

D

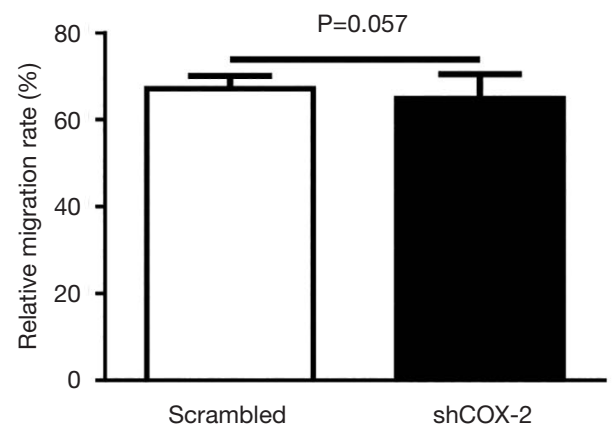

Figure 1 COX-2 knockdown has no effects on proliferation and migration of A431 cancer cells in vitro. A431 cells were infected with lentivirus expressing scrambled or COX-2 shRNA. (A) Cell lysates were subjected to western blot analysis with antibodies against COX-2 or internal control $\beta$-actin. (B) Growth curves of cancer cells with COX-2 knockdown were plotted over time, based on the cell numbers counted daily. Error bars represent standard deviations ( $\mathrm{n}=3, \mathrm{P}=0.489)$. $\mathrm{P}$ values were derived from the cell numbers at the end point. $(\mathrm{C}, \mathrm{D})$ COX-2 knockdown has no effect on cells migration in A431 cells, as evaluated by monolayer scratch assay. Representative microscopy images (magnification $\times 50$ ) of cells migration were shown in $(\mathrm{C}$ ), and the statistical results of cell migration was shown in (D). Error bars represent standard deviations ( $\mathrm{n}=2, \mathrm{P}=0.057)$. COX-2, cyclooxygenase 2 .

\section{COX-2 knockdown slightly inbibits proliferation and migration of SCC-13 cells in vitro}

We used another cell line SCC-13 to further confirm the effects of COX-2 on SCC cells in vitro. Western blot results demonstrated a successful knockdown of COX-2 expression in SCC-13 cancer cells (Figure 2A). Similarly, both cell growth and migration were only slightly suppressed in SCC-13 in vitro, as illustrated by growth curves over time and statistical results of cell monolayer scratch assay, respectively (Figure 2B,C,D). Both data from A431 and
SCC-13 demonstrate that COX-2 has only very limited direct effect on growth and migration of SCC cancer cells in vitro.

\section{COX-2 knockdown potently suppresses SCC growth in vivo}

Previous studies has shown that genetic knockout of COX-2 significantly suppresses SCC growth in vivo $(12,13)$. These results seems contrary to our in vitro data which showed no effects of disturbing COX-2 on growth of cancer cells. To confirm the function of COX-2 in vivo, we xenografted 
A
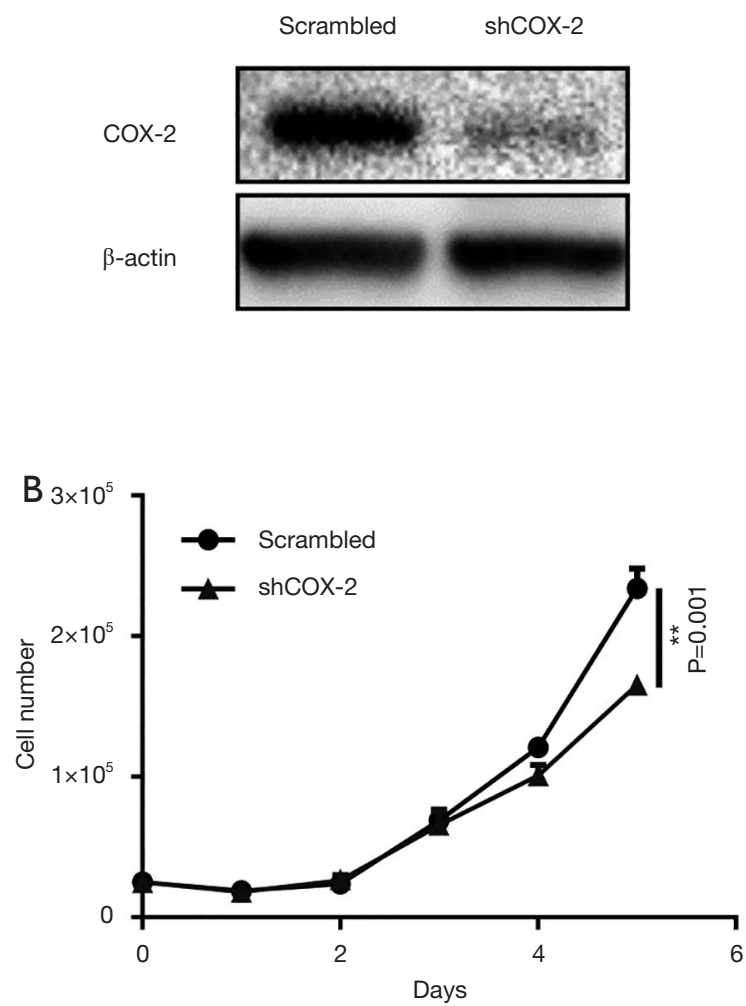

C

Scrambled
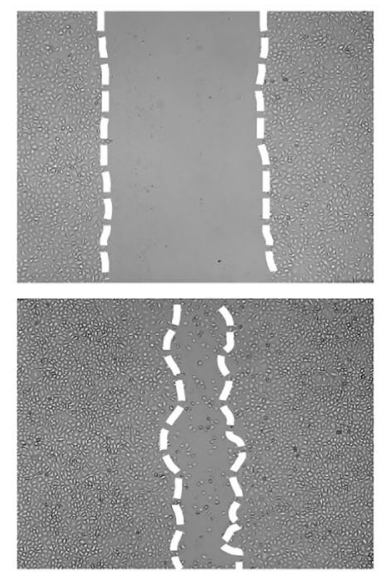

shCOX-2
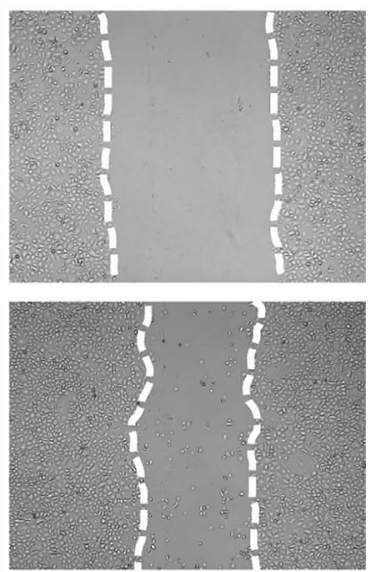

D

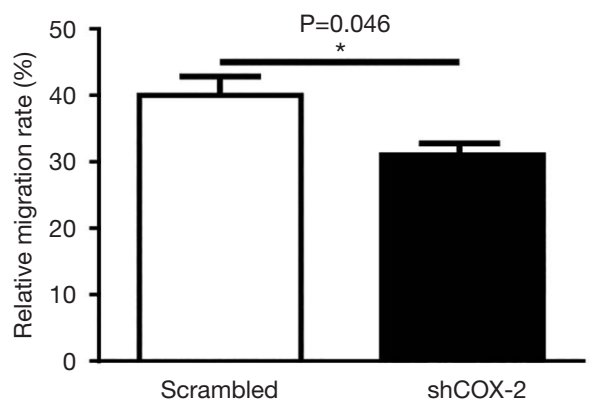

Figure 2 COX-2 knockdown slightly affects proliferation and migration of SCC-13 cancer cells in vitro. SCC-13 cells were infected with lentivirus expressing scrambled or COX-2 shRNA. (A) Cell lysates were subject to western blot analysis with antibodies against COX-2 or internal control $\beta$-actin. (B) Growth curves of SCC-13 with COX-2 knockdown were plotted over time, based on the cell numbers counted daily. Error bars represent standard deviations $(\mathrm{n}=3, \mathrm{P}=0.001)$. $\mathrm{P}$ values were derived from the cell numbers at the end point. (C,D) Cells migration in SCC-13 was evaluated by monolayer scratch assay. Representative microscopy images (magnification $\times 50$ ) of cells migration were shown in $(\mathrm{C})$, and the statistical results of cell migration was shown in (D). Error bars represent standard deviations ( $\mathrm{n}=2$, $\mathrm{P}=0.046)$. , $\mathrm{P}<0.05$; **, $\mathrm{P}<0.01$. COX-2, cyclooxygenase 2 ; SCC, squamous cell carcinoma.

cancer cells under the skin of nude mice (6-week-old healthy Balb/c female nude mice with body weight $16 \pm 1 \mathrm{~g}$, housed under SPF condition) and recorded tumor growth in vivo. In contrast to our in vitro results, COX-2 knockdown in both A431 and SCC-13 cells potently inhibited tumor growth in nude mice (Figure 3), as illustrated by tumor growth curves over time (Figure $3 A, E$ ) and tumor weight at the endpoint (Figure 3B,C,F,G). Western blot analysis confirmed COX-2 knockdown by shRNA, after cancer cells growing in tumors without drug selection pressure for more than 40 days (Figure 3D,H). These results confirmed that COX-2 plays pivotal role in SCC tumor growth in vivo.

\section{COX-2 knockdown inbibits proliferation of SCC cells in vivo}

COX-2 knockdown significantly suppresses tumor growth in vivo, but only slightly inhibits cancer cells growth in vitro. Proliferation of cancer cell might be suppressed by host environments, such as immune microenvironments, lacking of nutrition and nitrogen supplies, etc. We therefore examined whether proliferation of cancer cells were also inhibited by COX-2 knockdown in vivo. We injected $\mathrm{BrdU}$ into animal bearing tumors developed from A431 or SCC-13 cancer cells to label proliferative cells. Immunofluorescence staining of both proliferation 
A

A431

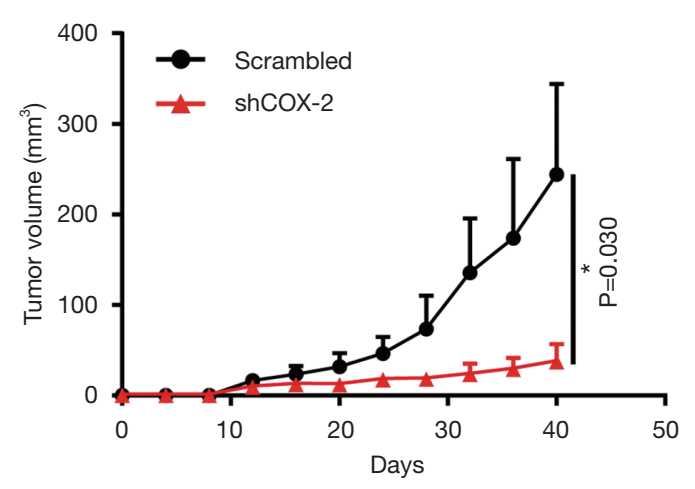

B

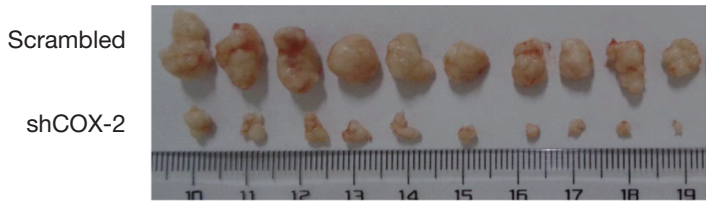

C

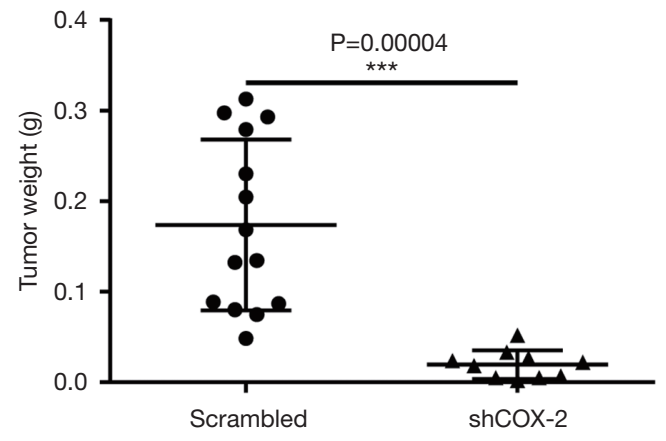

D

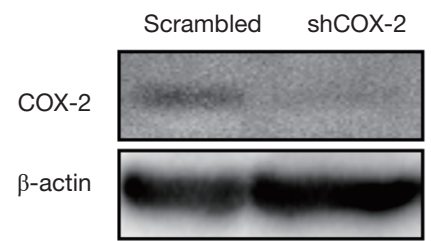

E

$\operatorname{scc13}$

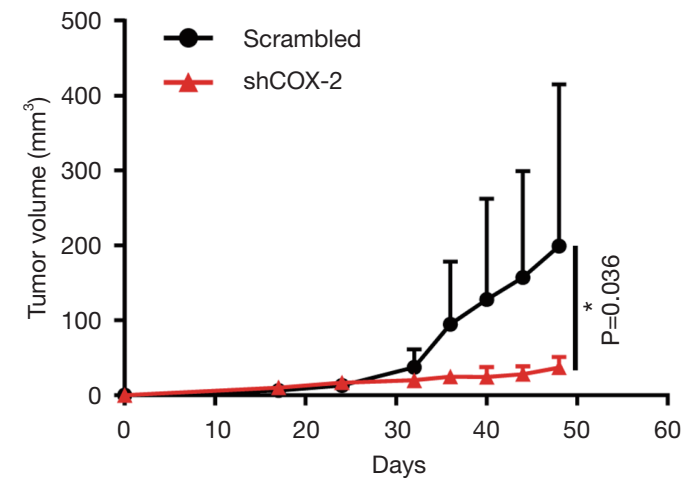

F

Scrambled

shCOX-2

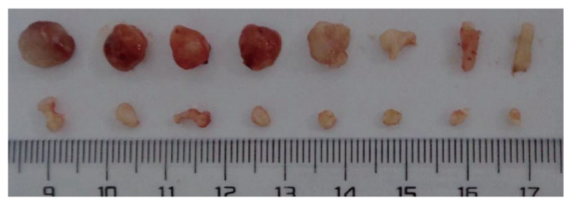

G

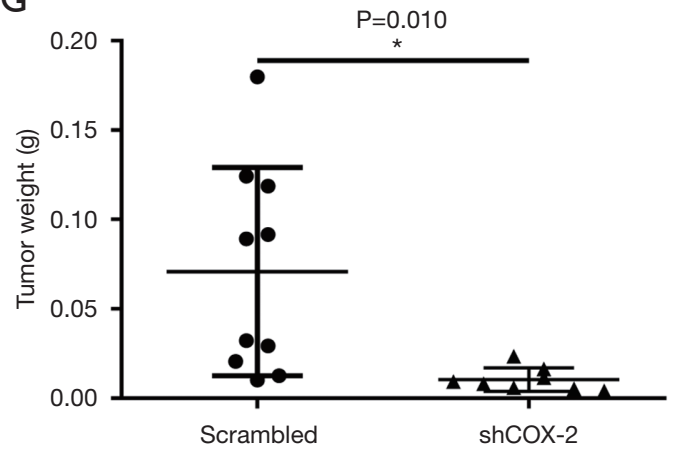

$\mathrm{H}$

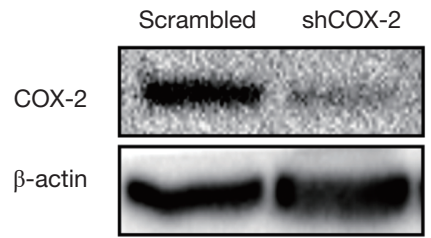

Figure 3 COX-2 knockdown potently suppresses tumor growth of human SCC cells in vivo. $1 \times 10^{6}$ A431 cells (A,B,C,D) or $2 \times 10^{6}$ SCC- 13 cells (E,F,G,H) infected with lentivirus expressing scrambled or COX-2 shRNA were inoculated subcutaneously together with matrigel into nude mice. $(\mathrm{A}, \mathrm{E})$ Tumor volumes were measured twice weekly and the tumor growth curves were plotted over time. $\mathrm{P}$ values were derived from the cell numbers at the end point. Error bars represent standard deviations (A431: n=10, $\mathrm{P}=0.030$; SCC-13: $\mathrm{n}=8, \mathrm{P}=0.036$ ). (B,F) Photographic illustration of 10 pairs of tumors harvested from nude mice at the end point (10 weeks). Each scale of the ruler represents $1 \mathrm{~mm}$. (C,G) Weights of tumors harvested from nude mice at the end point (A431: $\mathrm{n}=10, \mathrm{P}=0.00004$; SCC-13: $\mathrm{n}=8, \mathrm{P}=0.010$ ). Error bar represents standard deviations. (D,H) Immunoblots of COX-2 expressed in tumors from nude mice xenografted with A431 or SCC-13 cancer cells. ${ }^{*}, \mathrm{P}<0.05 ;{ }^{* * *}, \mathrm{P}<0.001$. COX-2, cyclooxygenase 2; SCC, squamous cell carcinoma. 

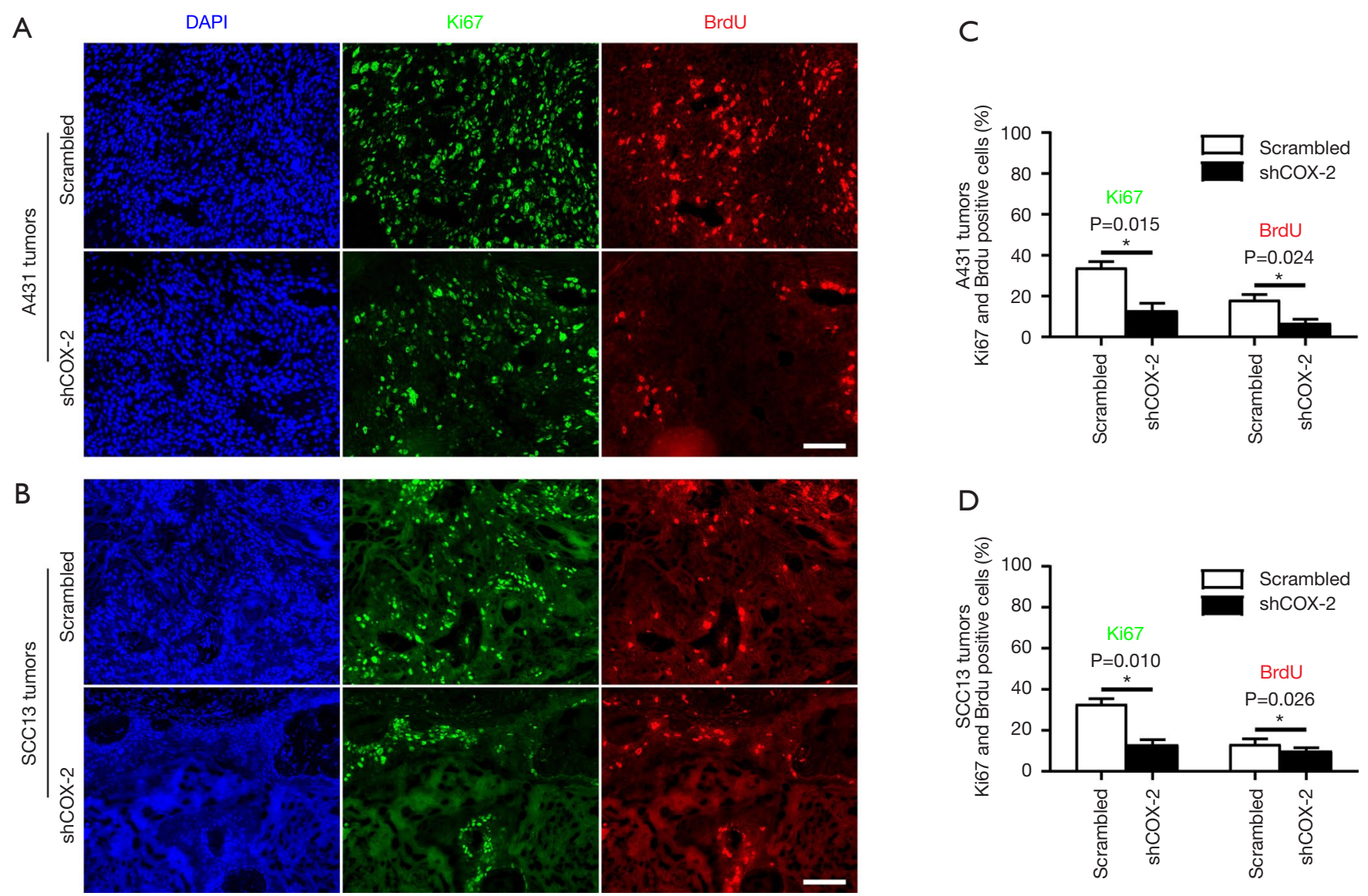

Figure 4 COX-2 knockdown suppresses proliferation of cancer cells in tumors. BrdU was intraperitoneally injected in nude mice bearing tumors developed from xenografted A431 or SCC-13 cancer cells to label proliferative cells. Tumor samples were harvested after 3 hours. $(\mathrm{A}, \mathrm{B})$ Both proliferation marker Ki67 and BrdU were immunofluorescence stained by specific antibodies. Scale bar, $100 \mu \mathrm{m}$. (C,D) Statistical results from (A,B). Error bar represents standard deviations $[(\mathrm{C}) \mathrm{n}=3, \mathrm{P}=0.015$ (Ki67) and $\mathrm{P}=0.024(\mathrm{BrdU}) ;(\mathrm{D}) \mathrm{n}=3, \mathrm{P}=0.010(\mathrm{Ki67})$ and $\mathrm{P}=0.026(\mathrm{BrdU})]$. *, $\mathrm{P}<0.05$. COX-2, cyclooxygenase 2; SCC, squamous cell carcinoma.

marker Ki67 and BrdU revealed that there were much less proliferative cancer cells in xenografted tumor samples with COX-2 knockdown (Figure 4). These results indicated that COX-2 might impact indirectly onto host microenvironments, instead of directly onto growth of cancer cells.

\section{COX-2 inbibitor celecoxib potently suppresses SCC growth in vivo}

Our results demonstrate that COX-2 could be a good target for treating SCC. However, previous reports only showed mild to modest inhibitory activity against SCC by COX-2 inhibitor. This might be due to inefficient drug administration route. To further examine the efficacy of targeting COX-2, we treated nude mice bearing xenografted SCC tumors by intraperitoneally injecting the COX-2 inhibitor celecoxib. As revealed by tumor growth curves (Figure 5A) or final tumor weights (Figure 5B,C), celecoxib significantly inhibited the tumor growth. The mice of treatment group and control group were similarly active, with similar body weights (data not shown), indicating the drugs had no obvious side effects at the experimental dosage. Immunofluorescence staining of both proliferation marker Ki67 and BrdU revealed that there were much less proliferative cancer cells in xenografted tumor samples treated with celecoxib (Figure 5D,E). These results demonstrate for the first time that celecoxib has potent anti-tumor activity against SCC through targeting COX-2. 
A

B
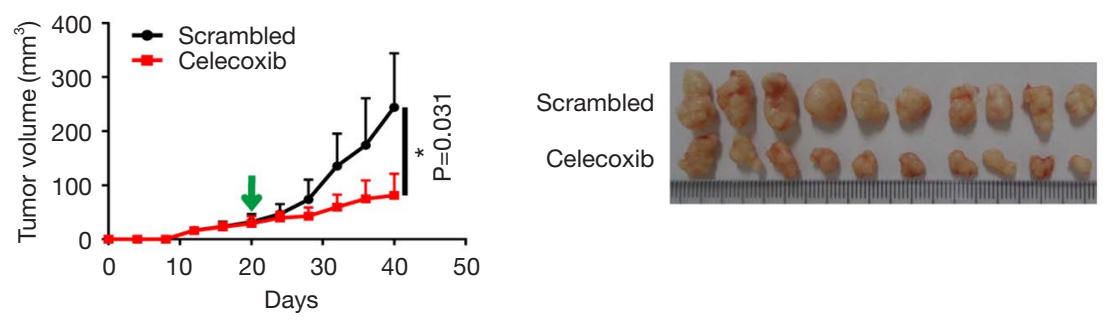

D
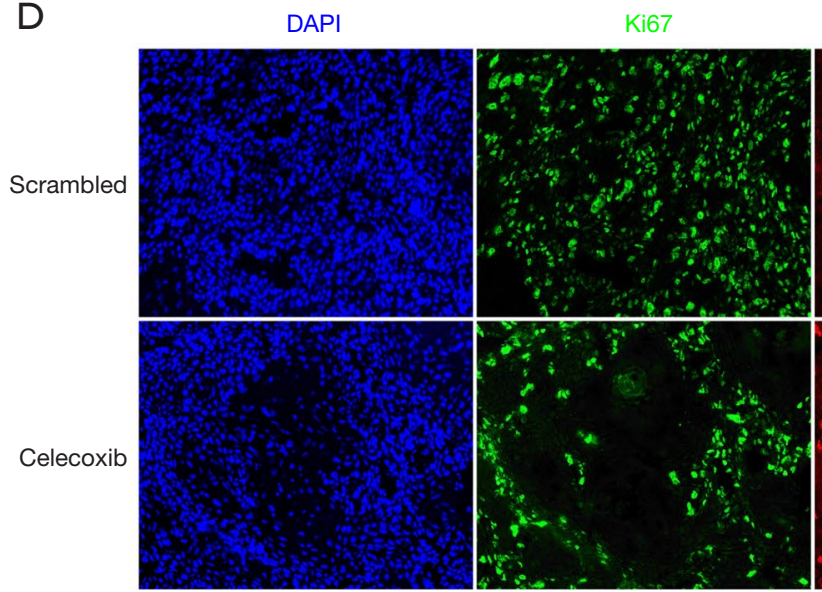

C

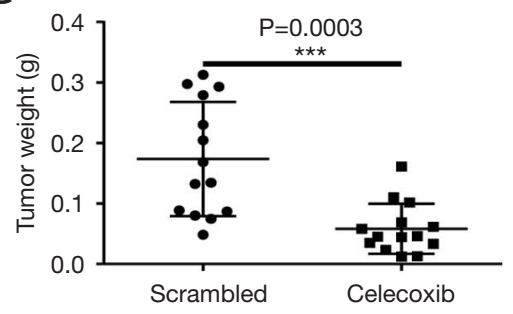

E

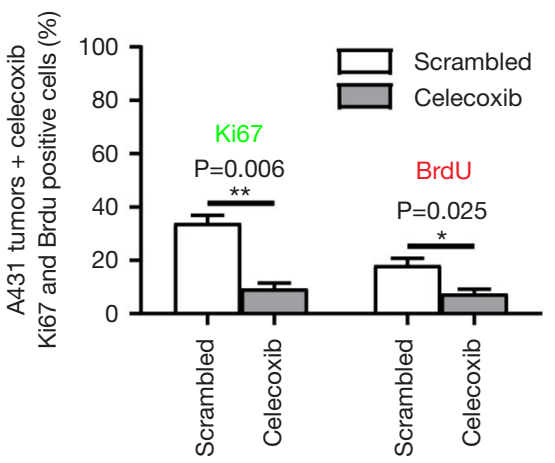

Figure 5 COX-2 inhibitor celecoxib potently inhibits SCC growth in mice. (A,B,C) $1 \times 10^{6} \mathrm{~A} 431$ cells together with matrigel were inoculated subcutaneously into nude mice. When tumors were obvious (tumor size $30-70 \mathrm{~mm}^{3}$ ), mice were randomly grouped to receive intraperitoneal injection with $5 \mathrm{mg} / \mathrm{kg}$ celecoxib or control solvent. The control group is the same as in Figure 3. (A) A431 tumor volumes were measured twice weekly and the tumor growth curves were plotted over time. Arrow points to the time point of the beginning of drug treatment. Error bars represent standard deviations ( $\mathrm{n}=10, \mathrm{P}=0.031$ ). (B) Photographic illustration of A431 tumor nodules harvested from nude mice at the end point. Each scale of the ruler represents $1 \mathrm{~mm}$. (C) Weights of A431 tumors harvested from nude mice at the end point. Error bars represent standard deviations ( $\mathrm{n}=10, \mathrm{P}=0.0003)$. ( $\mathrm{D}, \mathrm{E}) \mathrm{BrdU}$ was intraperitoneally injected in nude mice bearing tumors as above. Tumor samples were harvested after 3 hours. (D) Both proliferation marker Ki67 and BrdU were immunofluorescence stained by specific antibodies. Scale bar, $100 \mu \mathrm{m}$. (E) Statistical results from (D). Error bar represents standard deviations $[\mathrm{n}=3, \mathrm{P}=0.006$ (Ki67) and $\mathrm{P}=0.025$ (BrdU)]. *, $\mathrm{P}<0.05 ;$ **, $\mathrm{P}<0.01$; ***, $\mathrm{P}<0.001$. COX-2, cyclooxygenase 2; SCC, squamous cell carcinoma.

\section{Discussion}

In this work, we reported that knockdown of COX-2 only has slight or even no effect on proliferation of SCC cancer cells. However, in tumor xenograft nude mice model, both proliferation of cancer cells and growth of tumor tissues in vivo were significantly suppressed by COX-2 knockdown. In addition, we have shown that administration of COX-2 inhibitor celecoxib by intraperitoneal injection potently suppresses SCC growth in vivo. Our results demonstrate that targeting COX-2 represents a very promising therapeutic approach for treating SCC.

COX-2 is a PTGS2 enzyme responsible for generation of prostanoids like prostaglandin E2 (PGE2). COX-2 is rarely expressed in normal tissues, but significantly upregulated and promotes tumorigenesis in many types of cancers $(4,7)$. In other word, COX-2 is uniquely important for tumor growth but dispensable for normal tissues. Thus, COX-2 represents a promising target for treating cancers, since inhibiting its activity would presumably have little side effects.

Knockdown of COX-2 leads to growth arrest in hepatocellular carcinoma cells and pancreatic cancer cells in vitro $(20,21)$. However, in our results, knockdown of COX-2 only has very limited effect on proliferation of SCC 
cells in vitro, but has very dramatic inhibitory effect on proliferation of SCC cells as well as tumor growth in vivo. These results were confirmed in two different SCC cells lines, A431 and SCC-13. Therefore, our data argue that COX-2 promotes SCC tumorigenesis through indirect impact on tumor microenvironment instead of direct on cancer cells. Indeed, COX-2 and PGE2 have been reported to be released from cancer cells into microenvironment to block the activity for cytotoxic T lymphocytes (22), and COX-2 can induce IDO1 in cancer cells to suppresses host immunological responses (23). COX-2 is induced by inflammatory cytokines in cancer and further promote inflammation, therefore forming a reciprocal positive feedback to accelerate cancer progression $(24,25)$. It has been shown that a strong expression of COX-2 is induced in skin exposed to sunlight and SCC (8-10). In SCC, COX-2 is very likely involved in pre-inflammation during cancer progression.

Studies in transgenic mice has unambiguously demonstrated that COX-2 plays pivotal role in SCC tumorigenesis (11-13). However, COX-2 inhibitor only exhibited mild to moderate anti-cancer activity against SCC induced by UV $(14,15)$ This contradiction might be due to inefficient absorption of drug when it was incorporated in food and orally administrated. Therefore, we changed the drug administration route to examine the efficacy of the drug by intraperitoneal injection, and has demonstrated a very potent effects of COX-2 inhibitor on SCC growth.

\section{Conclusions}

COX-2 knockdown potently inhibits proliferation of cancer cells in vivo but not in vitro in SCC, indicating that COX-2 might impact on the interaction between cancer cells and surrounding microenvironments rather than on cancer cells directly. Our results confirm that both genetic and chemical inhibition of COX-2 exerts potent anti-cancer activity against SCC in vivo, demonstrating that targeting COX-2 is a very promising therapeutic approach for human SCC treatment.

\section{Acknowledgments}

Funding: This work was supported by the National Natural Science Foundation of China $(81972563,81903054)$ and the United Fujian Provincial Health and Education Project for Tackling the Key Research (WKJ2016-2-34).

\section{Footnote}

Reporting Checklist: The authors have completed the ARRIVE reporting checklist. Available at http://dx.doi. org/10.21037/tcr-20-3527

Data Sharing Statement: Available at http://dx.doi. org/10.21037/tcr-20-3527

Peer Review File: Available at http://dx.doi.org/10.21037/tcr20-3527

Conflicts of Interest: All authors have completed the ICMJE uniform disclosure form (available at http://dx.doi. org/10.21037/tcr-20-3527). The authors have no conflicts of interest to declare.

Ethical Statement: The authors are accountable for all aspects of the work in ensuring that questions related to the accuracy or integrity of any part of the work are appropriately investigated and resolved. The study was conducted in accordance with the Declaration of Helsinki (as revised in 2013). Experiments were performed under a project license (No. 2019033) granted by the Experimental Animal Ethics Committee of Shanghai University, in compliance with the guidelines of the Institutional Animal Care and Use Committee (IACUC).

Open Access Statement: This is an Open Access article distributed in accordance with the Creative Commons Attribution-NonCommercial-NoDerivs 4.0 International License (CC BY-NC-ND 4.0), which permits the noncommercial replication and distribution of the article with the strict proviso that no changes or edits are made and the original work is properly cited (including links to both the formal publication through the relevant DOI and the license). See: https://creativecommons.org/licenses/by-nc-nd/4.0/.

\section{References}

1. Apalla Z, Nashan D, Weller RB, et al. Skin cancer: epidemiology, disease burden, pathophysiology, diagnosis, and therapeutic approaches. Dermatol Ther (Heidelb) 2017;7:5-19.

2. Leiter U, Eigentler T, Garbe C. Epidemiology of skin cancer. Adv Exp Med Biol 2014;810:120-40.

3. Xie WL, Chipman JG, Robertson DL, et al. Expression of a mitogen-responsive gene encoding prostaglandin 
synthase is regulated by mRNA splicing. Proc Natl Acad Sci U S A 1991;88:2692-6.

4. Echizen K, Hirose O, Maeda Y, et al. Inflammation in gastric cancer: Interplay of the COX-2/prostaglandin E2 and Toll-like receptor/MyD88 pathways. Cancer Sci 2016;107:391-7. Erratum in: Cancer Sci 2016;107:1059.

5. Bakhle YS. COX-2 and cancer: a new approach to an old problem. Br J Pharmacol 2001;134:1137-50.

6. Subbaramaiah K, Dannenberg AJ. Cyclooxygenase 2: a molecular target for cancer prevention and treatment. Trends Pharmacol Sci 2003;24:96-102.

7. Hashemi Goradel N, Najafi M, Salehi E, et al. Cyclooxygenase-2 in cancer: a review. J Cell Physiol 2019;234:5683-99.

8. Buckman SY, Gresham A, Hale P, et al. COX-2 expression is induced by UVB exposure in human skin: implications for the development of skin cancer. Carcinogenesis 1998;19:723-9.

9. Wu Y, Liu H, Li J. Expression of p63 and cyclooxygenase-2 and their correlation in skin tumors. J Huazhong Univ Sci Technolog Med Sci 2007;27:206-8.

10. Kim KH, Park EJ, Seo YJ, et al. Immunohistochemical study of cyclooxygenase- 2 and $\mathrm{p} 53$ expression in skin tumors. J Dermatol 2006;33:319-25.

11. Muller-Decker K, Neufang G, Berger I, et al. Transgenic cyclooxygenase-2 overexpression sensitizes mouse skin for carcinogenesis. Proc Natl Acad Sci U S A 2002;99:12483-8.

12. Tiano HF, Loftin CD, Akunda J, et al. Deficiency of either cyclooxygenase (COX)-1 or COX-2 alters epidermal differentiation and reduces mouse skin tumorigenesis. Cancer Res 2002;62:3395-401.

13. Jiao J, Ishikawa TO, Dumlao DS, et al. Targeted deletion and lipidomic analysis identify epithelial cell COX-2 as a major driver of chemically induced skin cancer. Mol Cancer Res 2014;12:1677-88.

14. Fischer SM, Lo HH, Gordon GB, et al. Chemopreventive activity of celecoxib, a specific cyclooxygenase-2 inhibitor, and indomethacin against ultraviolet light-induced skin carcinogenesis. Mol Carcinog 1999;25:231-40.

15. Pentland AP, Schoggins JW, Scott GA, et al. Reduction

Cite this article as: Gao L, Wang TH, Chen CP, Xiang JJ, Zhao XB, Gui RY, Liao XH. Targeting COX-2 potently inhibits proliferation of cancer cells in vivo but not in vitro in cutaneous squamous cell carcinoma. Transl Cancer Res 2021;10(5):2219-2228. doi: $10.21037 /$ tcr-20-3527 of UV-induced skin tumors in hairless mice by selective COX-2 inhibition. Carcinogenesis 1999;20:1939-44.

16. Degen M, Barron P, Natarajan E, et al. RSK activation of translation factor eIF4B drives abnormal increases of laminin $\gamma 2$ and MYC protein during neoplastic progression to squamous cell carcinoma. PLoS One 2013;8:e78979.

17. Reagan-Shaw S, Nihal M, Ahmad N. Dose translation from animal to human studies revisited. FASEB J 2008;22:659-61.

18. Rheinwald JG, Beckett MA. Tumorigenic keratinocyte lines requiring anchorage and fibroblast support cultured from human squamous cell carcinomas. Cancer Res 1981;41:1657-63.

19. Han H, Yang S, Lin SG, et al. Effects and mechanism of downregulation of COX-2 expression by RNA interference on proliferation and apoptosis of human breast cancer MCF-7 cells. Mol Med Rep 2014;10:3092-8.

20. Lv X, Chen Z, Li S, et al. Knockdown of cyclooxygenase-2 leads to growth inhibition and cell cycle arrest in hepatocellular carcinoma cells. Onco Targets Ther 2019;12:4341-9.

21. Li W, Mao Z, Fan X, et al. Cyclooxygenase 2 promoted the tumorigenecity of pancreatic cancer cells. Tumour Biol 2014;35:2271-8.

22. Miao J, Lu X, Hu Y, et al. Prostaglandin E2 and PD-1 mediated inhibition of antitumor CTL responses in the human tumor microenvironment. Oncotarget 2017;8:89802-10.

23. Hennequart M, Pilotte L, Cane S, et al. Constitutive IDO1 Expression in Human Tumors Is Driven by Cyclooxygenase- 2 and Mediates Intrinsic Immune Resistance. Cancer Immunol Res 2017;5:695-709.

24. Desai SJ, Prickril B, Rasooly A. Mechanisms of Phytonutrient Modulation of Cyclooxygenase-2 (COX-2) and Inflammation Related to Cancer. Nutr Cancer 2018;70:350-75.

25. Hosseini F, Mahdian-Shakib A, Jadidi-Niaragh F, et al. Anti-inflammatory and anti-tumor effects of $\alpha-1-$ guluronic acid (G2013) on cancer-related inflammation in a murine breast cancer model. Biomed Pharmacother 2018;98:793-800. 